\title{
Article
}

\section{Urinary excretion of 8-0xo-7, 8-dihydro-2'-deoxyguanosine as a predictor of the development of diabetic nephropathy}

\author{
Y. Hinokio ${ }^{1}$, S. Suzuki ${ }^{1,2}$, M. Hirai ${ }^{1}$, C. Suzuki ${ }^{1}$, M. Suzuki ${ }^{2}$, T. Toyota ${ }^{1}$ \\ ${ }^{1}$ Department of Molecular Metabolism and Diabetes, Tohoku University, Graduate School of Medicine, Sendai, Japan \\ 2 Department of Life Science, Shoukei Women's Junior Collage, Natori, Japan
}

\begin{abstract}
Aims/hypothesis. The increased oxidative stress in diabetes is known to contribute to the progression of diabetes and its complications. We have reported a significant relation between the content of 8-oxo-7,8-dihydro-2'-deoxyguanosine (8-oxodG), a product of oxidative DNA damage in urine or leukocytes and the severity of diabetic nephropathy and retinopathy [1]. We investigated whether 8-oxodG in urine or leukocytes is associated with the progression of diabetic nephropathy.

Methods. We measured urinary 8-oxodG contents at entry and carried out a prospective longitudinal study to assess the progression of nephropathy over 5 years. Results. There was a significant progression of diabetic nephropathy in the patients with higher excretion of 8-oxodG in urine compared with the patients with moderate or lower excretion of 8-oxodG. There
\end{abstract}

was no significant association between the leukocyte 8-oxodG contents and the development of nephropathy. The multivariate logistic regression analysis suggests that the urinary 8-oxodG was the strongest predictor of nephropathy among several known risk factors.

Conclusion/interpretation. This study provides evidence that increased oxidative stress has a primary role in the pathogenesis of diabetic nephropathy. A local enhancement of oxidative stress in diabetic kidney might explain the possible linkage between the increased urinary excretion of 8-oxodG and the development of nephropathy. 8-oxodG in urine is a useful clinical marker to predict the development of diabetic nephropathy in diabetic patients. [Diabetologia (2002) 45:877-882]

Keywords Oxidative stress, 8-oxo-7, 8-dihydro-2'deoxyguanosine, reactive oxygen species, diabetic nephropathy.
The link between hyperglycaemia and the complications of diabetes is still not clear. Increased oxidative stress in diabetes might contribute to the pathogenesis of diabetic complications, including nephropathy $[2$,

Received: 26 November 2001 / Revised: 12 February 2002

Published online: 8 May 2002

(C) Springer-Verlag 2002

Corresponding author: S. Suzuki, MD, PhD, Department of Molecular Metabolism and Diabetes, Tohoku University, Graduate School of Medicine, Seiryou-machi 1-1, Aoba-ku, Sendai 980-8574, Japan, e-mail: ssuzuki@int3.med.tohoku.ac.jp Abbreviations: 8-oxodG , 8-oxo-7, 8-Dihydro-2' -deoxyguanosine; dG, deoxyguanosine; ROS, reactive oxygen species; $\mathrm{PKC}$, protein kinase $\mathrm{C}$; Norm, normoalbuminuria; Micro, microalbuminuria; Macro, macroalbuminuria
3]. Oxidative stress is one of the important mediators of vascular complications in diabetes including nephropathy. The hyperglycaemia-induced generation of reactive oxygen species (ROS) is linked by three pathological pathways to diabetic complications: activation of protein kinase $\mathrm{C}$ (PKC), the formation of advanced glycation end products (AGE), and sorbitol accumulation [4, 5]. Normalising mitochondrial ROS production was reported to block these hyperglycaemia-mediated signalling pathways.

8-oxodG is a product of oxidative DNA damage following specific enzymatic cleavage after ROSinduced 8-hydroxylation of the guanine base in mitochondrial and nuclear DNA [6]. 8-oxodG is known to be a sensitive marker of oxidative DNA damage and of the total systemic oxidative stress in vivo [7]. Sev- 
eral reports described an increase in the 8-oxodG content in mononuclear cells and urine in Type I and Type II (non-insulin-dependent) diabetic patients $[8,9,10]$. We have reported a significant relation between the urinary or leukocyte 8 -oxodG or both and the severity of diabetic nephropathy and retinopathy [1].

To clarify the contribution of oxidative DNA damage in the diabetic kidney to the development of diabetic nephropathy, we measured the urinary and leukocyte 8-oxodG contents at entry and carried out a prospective study to assess the progression of nephropathy after 5 years. This study provides direct evidence that increased urinary 8-oxodG at entry was associated with the development of diabetic nephropathy after 5 years. 8-oxodG contents in urine can be used as a predictor for the progression of diabetic nephropathy. We discussed the possible contribution of urinary 8-oxodG to the development of diabetic nephropathy.

\section{Subjects and methods}

Protocol. We conducted a prospective longitudinal study of Type II diabetic patients with normoalbuminuria or microalbuminuria. The study protocol was reviewed and approved by the Tohoku University Institutional Review Board. Informed consent was obtained from each patient. The study cohort was drawn from a cross-sectional study of diabetic patients who attended diabetes clinics at the Tohoku University Hospital in 1994 and 1995. 532 Japanese non-obese and non-smoking diabetic subjects from the initial cohort were included in the 5-year follow-up study, after diabetic patients with other kidney diseases were omitted. Based on the results of the urinary albumin-to-creatinine ratio in $24 \mathrm{~h}$ urine samples, diabetic patients were classified as normoalbuminuria or microalbuminuria. Normoalbuminuria, microalbuminuria and macroalbuminuria were defined as having a urinary albumin-to-creatinine ratio below $15,15-200$ and above $200 \mu \mathrm{g} / \mathrm{g}$ creatinine $(\mathrm{Cr}) / 24 \mathrm{~h}$, respectively. Diabetic patients received usual clinical care in the diabetes clinics at the Tohoku University Hospital during the 5-year follow-up period. After the 5-year-followup, 24-h overnight urine samples were collected and analysed for albumin and creatinine.

We analysed 396 Japanese non-obese and non-smoking Type II diabetic patients with normoalbuminuria $(<15 \mu \mathrm{g} / \mathrm{g}$ creatinine $(\mathrm{Cr}) / 24 \mathrm{~h}$; normo-group; $n=115)$ or microalbuminuria (15-200 $\mu \mathrm{g} / \mathrm{g} \mathrm{Cr} / 24 \mathrm{hr}$, micro-group; $n=281)$ after 5-years follow-up study.

The diabetic patients were classified into three subgroups according to the degree of $24 \mathrm{~h}$ urinary excretion of 8-oxodG at entry: the groups of low (L1 group: below $200 \mathrm{pmol} / \mathrm{Kg} /$ day; $n=123$ ), moderate (M1 group: $200-400 \mathrm{pmol} /$ $\mathrm{Kg} /$ day; $n=173$ ), and high excretion (H1 group: above $400 \mathrm{pmol} / \mathrm{Kg} /$ day; $n=100)$. These three groups had similar entry contents of blood pressure, $\mathrm{HbA}_{1 c}$, urinary albumin excretion rates, and other clinical parameters, as shown in Table 1.

The diabetic patients were also classified into three other subgroups according to the contents of 8-oxodG in leukocytes at entry: the groups of low (L2 group: below $60 \mathrm{fmol} / \mu \mathrm{g}$ DNA; $n=139$ ), moderate (M2 group: $60-80 \mathrm{fmol} / \mu \mathrm{g}$ DNA; $n=158$ ), and high contents (H2 group: above $80 \mathrm{fmol} / \mu \mathrm{g}$ DNA; $n=109$ ) in leukocytes. There was no difference in the entry contents of blood pressure, $\mathrm{HbA}_{1 \mathrm{c}}$, urinary albumin excretion rates, and other clinical parameters among the three groups (Data not shown).

The 8-oxodG samples from urine and leukocytes were prepared and analysed by high performance liquid chromatography with electro-chemical detection (HPLC-ECD) [10]. The urinary albumin concentration was measured by an enzymelinked immunosorbent assay.

Statistical analysis. All measurements are presented as means \pm SD. Statistical difference was assessed by a repeated measure ANOVA. Wilcoxon's test was used for paired comparison, and the Mann-Whitney U-test for unpaired comparison. Multivariate logistic regression analysis was conducted using SPSS 10.0 for windows. Statistical significance was accepted at the $95 \%$ confidence level $(p<0.05)$.

Table 1. Baseline clinical and biochemical characteristics of the Type II diabetic patients

Urine 8-oxodG excretion (pmol/Kg/day)

\begin{tabular}{lccc} 
& $\mathrm{L}_{1}$ group $(<200)$ & $\mathrm{M}_{1}$ group (200 400) & $\mathrm{H}_{1}$ group (>400) \\
\hline$n$ & 123 & 173 & 100 \\
Men (\%) & 47 & 48 & 48 \\
Age at onset of diabetes (years) & $51.4 \pm 11.4$ & $52.0 \pm 9.8$ & $52.2 \pm 11.1$ \\
Duration (years) & $9.3 \pm 8.2$ & $9.4 \pm 10.1$ & $23.7 \pm 7.7$ \\
BMI & $22.7 \pm 5.1$ & $23.7 \pm 4.1$ & $7.5 \pm 2.9$ \\
HbA $(\%)$ & $7.3 \pm 2.6$ & $1.25 \pm 0.47$ & $1.12 \pm 0.77$ \\
Triglyceride (mmol/l) & $1.27 \pm 0.55$ & $5.10 \pm 1.02$ & $4.98 \pm 1.40$ \\
Total cholesterol (mmol/l) & $5.09 \pm 1.32$ & $1.31 \pm 0.41$ & $1.30 \pm 0.41$ \\
HDL cholesterol (mmol/l) & $1.34 \pm 0.41$ & $126 \pm 19$ & $130 \pm 23$ \\
Systolic blood pressure (mmHg) & $125 \pm 18$ & $80 \pm 18$ & $80 \pm 20$ \\
Diastolic blood pressure (mmHg) & $77 \pm 15$ & 30 & 31 \\
Therapy for hypertension $(\%)$ & 27 & $105 \pm 15$ & $104 \pm 14$ \\
Creatinine clearance (ml/min) & $105 \pm 12$ & 17 & 18 \\
Retinopathy (\%) & 16 & 26 & 30 \\
Current smokers $(\%)$ & 27 & $24 / 38 / 38$ & $25 / 36 / 39$
\end{tabular}

Means \pm SD 


\section{Results}

After the 5-year prospective study, we confirmed a progression of diabetic nephropathy in the normo-H1 group as compared with the normo-L1 group $(p<0.002)$ and normo-M1 group $(p<0.002)$ (Table 2). In the micro-group, the incidence of macroalbuminuria (>200 $\mu \mathrm{g} / \mathrm{g} \mathrm{Cr} / 24 \mathrm{~h}$ ) was increased in the micro-H1 group as compared with the micro-L1 group $(p<0.0001)$ and the micro-M1 group $(p<0.0001)$ (Table 2). There was a correlation between the 8 -oxodG content in urine and leukocytes $(r=0.425$, $p<0.05$ ) (data not shown). However, there was no significant association between the leukocyte 8 -oxodG contents and the development of nephropathy (Table 3).

Table 2. The progression of diabetic nephropathy in the Type II diabetic patients after 5 years. Classification with urinary excretion of 8-oxodG at entry

\begin{tabular}{|c|c|c|c|c|}
\hline \multicolumn{2}{|c|}{ Nephropathy } & \multicolumn{3}{|c|}{ Urine 8-oxodG Excretion (pmol/Kg/day) } \\
\hline At entry & After 5 years & L1 group $(<200)$ & M1 group (200 400) & H1 group $(>400)$ \\
\hline Norm & $\begin{array}{l}\text { Norm } \\
\text { Micro } \\
\text { Macro }\end{array}$ & $\begin{array}{c}26(76) \\
8(24) \\
0(0)\end{array}$ & $\begin{array}{c}36(71) \\
15(29) \\
0(0)\end{array}$ & $\begin{array}{c}11(37) \\
17(57) \\
2(7)\end{array}$ \\
\hline \multicolumn{2}{|c|}{$p$ value (vs $\mathrm{H}$ group) } & $p=0.001$ & $p=0.002$ & \\
\hline Micro & $\begin{array}{l}\text { Micro } \\
\text { Macro }\end{array}$ & $\begin{array}{l}73(82) \\
16(18)\end{array}$ & $\begin{array}{l}91(75) \\
31(25)\end{array}$ & $\begin{array}{l}28(40) \\
42(60)\end{array}$ \\
\hline \multicolumn{2}{|c|}{$p$ value (vs $\mathrm{H}$ group) } & $p<0.0001$ & $p<0.0001$ & \\
\hline
\end{tabular}

Data $\operatorname{are} n(\%)$

Table 3. The development of diabetic nephropathy after 5 years: the effect of classification with the content of 8-oxodG in leukocytes at entry

\begin{tabular}{|c|c|c|c|c|}
\hline \multicolumn{2}{|c|}{ Nephropathy } & \multicolumn{3}{|c|}{ Leukocyte 8-oxodG Content (fmol/ $\mu \mathrm{g}$ DNA) } \\
\hline At entry & After 5 years & L2 group $(<60)$ & M2 group (60 80) & $\mathrm{H} 2$ group $(>80)$ \\
\hline \multirow[t]{3}{*}{ Norm } & Norm & $26(68)$ & $39(67)$ & $18(53)$ \\
\hline & Micro & $11(29)$ & $14(33)$ & $15(44)$ \\
\hline & Macro & 1 (3) & $0(0)$ & $1(3)$ \\
\hline \multirow[t]{2}{*}{ Micro } & Micro & $73(82)$ & $91(75)$ & $28(40)$ \\
\hline & Macro & $16(18)$ & $31(25)$ & $42(60)$ \\
\hline
\end{tabular}

Data are $n(\%)$

Table 4 The duration-adjusted variables for the development of diabetic nephropathy

\begin{tabular}{lccc}
\hline & \multicolumn{2}{l}{ Progression } & \multirow{2}{*}{$p$} \\
\cline { 2 - 3 } Variables & Yes $(n=131)$ & No $(n=265)$ & \\
\hline Men (\%) & 44 & 50 & NS \\
Age at diagnosis of diabetes (years) & $51.7 \pm 9.6$ & $51.9 \pm 10.7$ & NS \\
Family history of diabetes (\%) & 51 & 43 & NS \\
BMI & $23.7 \pm 4.5$ & $22.8 \pm 4.0$ & NS \\
Systolic blood pressure (mmHg) & $136 \pm 22$ & $127 \pm 22$ & 0.00015 \\
Diastolic blood pressure (mmHg) & $85 \pm 19$ & $78 \pm 17$ & 0.00024 \\
Taking ACE inhibitors (\%) & 20 & 23 & NS \\
HbAlc (\%) & $7.5 \pm 0.9$ & $7.3 \pm 0.9$ & 0.038 \\
8-oxodG in urine (pmol//Kg/day) & $391 \pm 109$ & $341 \pm 76$ & $<0.00001$ \\
8-oxodG in leukocytes(fmol/mg DNA) & $77 \pm 18$ & $75 \pm 16$ & NS \\
Fasting triglycerides (mmol/l) & $1.26 \pm 0.5$ & $1.20 \pm 0.5$ & NS \\
Total Cholesterol (mmol/l) & $5.2 \pm 0.5$ & $5.0 \pm 0.5$ & NS \\
HDL cholesterol (mmol/l) & $1.28 \pm 0.2$ & $1.34 \pm 0.2$ & NS \\
Current smokers (\%) & 32 & 24 & NS \\
Diet / OHA / Insulin (\%) & $27 / 36 / 37$ & $23 / 39 / 39$ & \\
\hline
\end{tabular}

Means \pm SD 


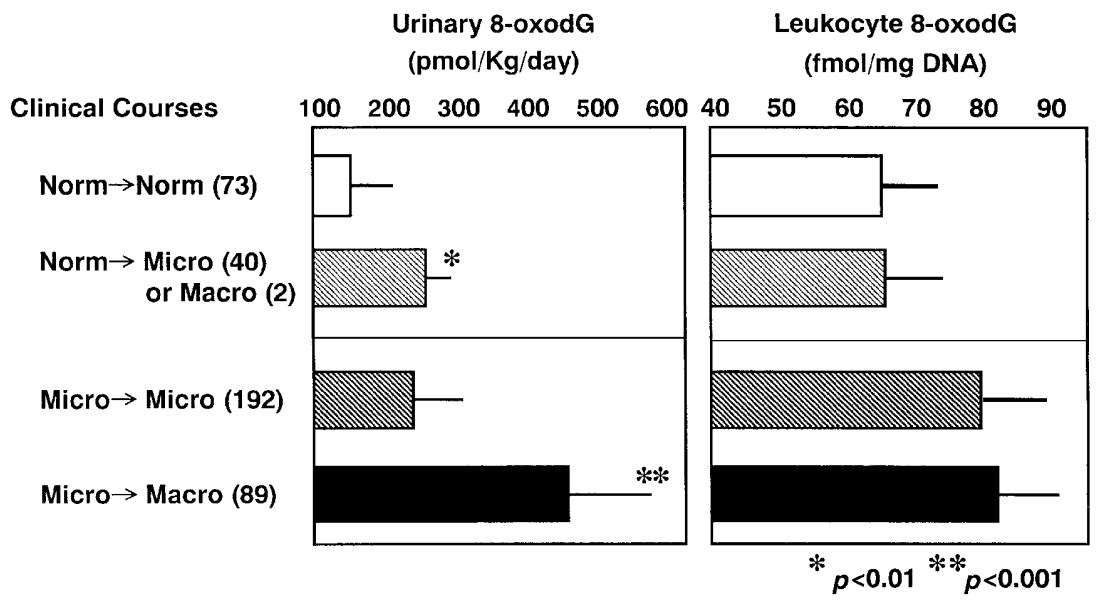

Fig. 1. The contents of 8-oxodG in urine and leukocytes in the Type II diabetic patients with or without the progression of diabetic nephropathy

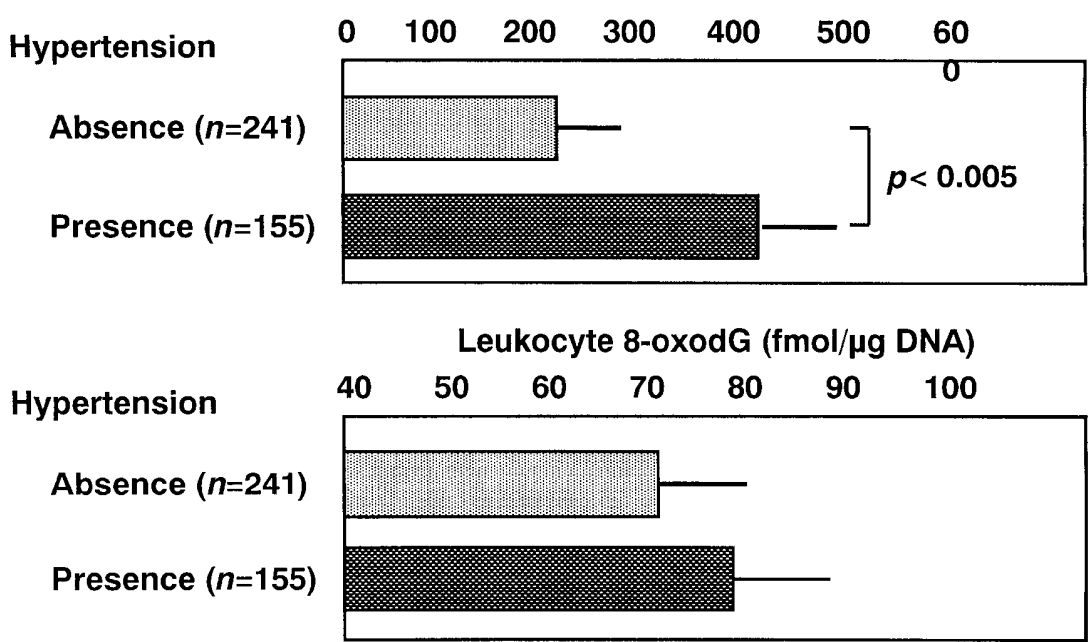

Fig. 2. The contents of 8 -oxodG in urine and leukocytes in the Type II diabetic patients with or without hypertension at entry

Table 5 Multivariate logistic regression analysis of variables for the prediction of diabetic nephropathy

\begin{tabular}{lll}
\hline Variable & $\begin{array}{l}\text { Standardized relative risk } \\
(95 \%-\mathrm{CI})\end{array}$ & $p$ \\
\hline Urinary 8-oxodG & $2.71(1.78-3.88)$ & 0.0001 \\
Duration of diabetes & $1.24(1.01-1.59)$ & 0.04 \\
Systolic blood pressure & $1.73(1.32-2.20)$ & 0.01 \\
Diastolic blood pressure & $1.93(1.52-2.64)$ & 0.0005 \\
HbA $_{1 \mathrm{c}}$ & $1.32(1.07-1.61)$ & 0.02 \\
\hline
\end{tabular}

The risk factors for the development of nephropathy were the duration of diabetes, $\mathrm{HbA}_{1 \mathrm{c}}$, blood pressure and urinary excretion of 8-oxodG. After adjusting for the diabetes duration, $\mathrm{HbA}_{1 \mathrm{c}}(7.5 \pm 0.9$ vs $7.3 \pm 0.9$, $p=0.038$ ) blood pressure (systolic: $136 \pm 22$ vs $127 \pm 22$, $p=0.00015$; diastolic; $85 \pm 19$ vs $78 \pm 17, p=0.00024$ ), and urinary 8 -oxodG $(391 \pm 109$ vs $341 \pm 76$, $p<0.00001)$ were higher in the progression group than in the non-progression group (Table 4). The progression group had higher 8-oxodG contents in urine than the non-progression group $(p<0.005)$ (Fig. 1). However, there were no differences in the 8-oxodG contents in leukocytes between the progression group and the non-progression group. There were no differences in the taking of angiotensin-converting enzyme inhibitors between the progression group and the non-progression group. There was a weak association between the urinary excretion of 8 -oxodG and average $\mathrm{HbA}_{1 \mathrm{c}}$ $(p<0.05)$. There was no significant difference between the content of 8-oxo-dG in urine or leukocytes and the development of retinopathy or neuropathy, respectively.

In multivariate logistic regression analysis, higher urinary excretion of 8-oxodG, a longer duration of diabetes, higher systolic and diastolic blood pressures, and $\mathrm{HbA}_{1 \mathrm{c}}$ were independently associated with the development of nephropathy (Table 5 ). The urinary 8-oxodG was the strongest predictor of nephropathy among the several known risk factors for the develop- 
ment of nephropathy, ie. diabetes duration, $\mathrm{HbA}_{1 \mathrm{c}}$, and hypertension.

Urinary 8-oxodG was higher in the patients with hypertension than in those without hypertension $(p<0.005)$ (Fig. 2). However, there was no difference in the leukocyte 8-oxodG contents between the patients with hypertension than in those without hypertension.

\section{Discussion}

The Diabetes Control and Complications Trial (DCCT), UK Prospective Diabetes Study (UKPDS), and the Kumamoto study established a causal relationship between chronic hyperglycaemia and diabetic microvascular diseases. Hyperglycaemia generates ROS and attenuates anti-oxidative mechanisms by scavenging enzymes and antioxidant substances [2, 3]. Oxidative stress is one of the important mediators of diabetic vascular complications including nephropathy [3].

8-oxodG is known as a sensitive biomarker of oxidative DNA damage $[5,6]$. We have reported that the contents of 8-oxodG in urine and in the leukocytes were associated with the severity of diabetic nephropathy as well as diabetic retinopathy in a cross-sectional study of Japanese diabetic patients [1]. In our study, the risk factors for the development of nephropathy were the duration of diabetes, $\mathrm{HbA}_{1 \mathrm{c}}$, blood pressure, and urinary excretion of 8-oxodG. The urinary 8-oxodG was the strongest predictor of nephropathy among these independent risk factors.

These data suggest that increased oxidative stress has a primary role in the pathogenesis of diabetic nephropathy and that increased oxidative stress is not a secondary indicator of end-stage tissue damage in the diabetic kidney.

We have reported that urinary excretion of 8-oxodG was significantly correlated with the 8-oxodG content in the leukocytes in Japanese diabetic patients [1] but this study could not confirm a significant relation between the development of nephropathy and the content of 8-oxodG in leukocytes. A local enhancement of oxidative stress in the diabetic kidney might explain the possible linkage between the increased urinary excretion of 8-oxodG and the development of nephropathy.

Recent reports showed that oxidative stress was also involved in hypertension and that urinary 8-oxodG was increased in patients with hypertension and in hypertensive animal models $[11,12,13,14]$. We speculate that the increased urinary 8-oxodG in the diabetic patients might have resulted not only from the increased systemic oxidative stress, but also locally from the kidney, where both hypertension and hyperglycaemia enhance the oxidative stress. Treatment with resveratrol had protective effects on the oxidative damage in stroke-prone spontaneously hypertensive rats [15].
Evidence suggests that genetic factors might contribute to the pathogenesis and progression of diabetic nephropathy. A familial predisposition to oxidative stress is reported in Type I diabetic patients [16]. An association has been found between the genetic polymorphism of paraoxonase enzyme and oxidative DNA damage in patients with Type II diabetes mellitus and in control subjects [17]. The genetic polymorphism of paraoxonase or other genes might provide a possible linkage between the familial susceptibility to nephropathy and the familial predisposition of oxidative DNA damages.

This suggests that hyperglycaemia, hypertension and other genetic factors might promote the oxidative DNA damages in kidney and accelerate the progress of diabetic nephropathy. Treatment with antioxidants is thought to improve the hyperglycaemia-attenuated anti-oxidative mechanisms by scavenging enzymes and antioxidant substances $[2,3]$. In animal models, diabetic nephropathy was successfully improved or ameliorated by a treatment with anti-oxidants, such as taurine, vitamin $\mathrm{E}$ and alpha-lipoic acid $[18,19]$. In a human clinical study, high-dose vitamin E supplementation normalizes creatinine clearance in patients with Type I diabetes [20]. Treatment with alpha-lipoic acid also improved the progression of endothelial cell damage and albuminuria in diabetic patients [21]. This study provides further evidence that anti-oxidant therapy has essential therapeutic implications for the prevention of diabetic nephropathy.

In conclusion, we have shown that 8-oxodG could be a useful specific indicator pointing to the onset and progression of diabetic nephropathy.

Acknowledgements This investigation was supported partly by a grant from Japan Diabetes Foundation and Grant-in-Aids for the Ministry of Education, Science, Sports and Culture of Japan (12671194). We thank C. Suzuki for technical assistance.

\section{References}

1. Giugliano D, Ceriello A, Paolisso G (1996) Oxidative stress and diabetic vascular complications. Diabetes Care 19:257-267

2. Baynes JW, Thorpe SR (1999) Role of oxidative stress in diabetic complications. A new perspective on an old paradigm. Diabetes 48:1-9

3. Nishikawa T, Edelstein D, Du XL et al. (2000) Normalizing mitochondrial superoxide production blocks three pathways of hyperglycemic damage. Nature 404:787-790

4. Nishikawa T, Edelstein D, Brownlee M (2000) The missing link: a single unifying mechanism for diabetic complications. Kidney Int [Suppl 77]:S26-S30

5. Loft S, Poulsen HE (1999) Markers of oxidative damage to DNA: antioxidants and molecular damage. Methods Enzymol 300:166-184

6. Cooke MS, Evans MD, Herbert KE, Lunec J (2000) Urinary 8-oxo-2'-deoxyguanosine-source, significance and supplements. Free Radic Res 32:381-397 
7. Ha H, Kim C, Son Y, Chung MH, Kim KH (1994) DNA damage in the kidneys of diabetic rats exhibiting microalbuminuria. Free Radic Biol Med 16:271-274

8. Dandona P, Thusu K, Cook S et al. (1996) Oxidative damage to DNA in diabetes mellitus. Lancet 347:444-445

9. Leinonen J, Lehtimaki T, Toyokuni S et al. (1997) New biomarker evidence of oxidative DNA damage in patients with non-insulin-dependent diabetes mellitus. FEBS Lett 417:150-152

10. Hinokio Y, Suzuki S, Hirai M, Chiba M, Hirai A, Toyota T (1999) Oxidative DNA damage in diabetes mellitus: its association with diabetic complications. Diabetologia 42: 995-998

11. Negishi H, Ikeda K, Sagara M, Sawamura M, Yamori Y (1999) Increased oxidative DNA damage in stroke-prone spontaneously hypertensive rats. Clin Exp Pharmacol Physiol 26:482-484

12. Negishi H, Njelekela M, Ikeda K et al. (2000) Assessment of in vivo oxidative stress in hypertensive rats and hypertensive subjects in Tanzania, Africa. Hypertens Res 23: 285-289

13. Mizutani K, Ikeda K, Nishikata T, Yamori Y (2000) Phytoestrogens attenuate oxidative DNA damage in vascular smooth muscle cells from stroke-prone spontaneously hypertensive rats. J Hypertens 18:1833-1840

14. Negishi H, Ikeda K, Kuga S et al. (2000) The relation of oxidative DNA damage to hypertension and other cardiovascular risk factors in Tanzania. J Hypertens 19:529-533
15. Mizutani K, Ikeda K, Kawai Y, Yamori Y (2001) Protective effect of resveratrol on oxidative damage in male and female stroke-prone spontaneously hypertensive rats. Clin Exp Pharmacol Physiol 28:55-59

16. Matteucci E, Giampietro O (2000) Oxidative stress in families of type 1 diabetic patients. Diabetes Care 23: 1182-1186

17. Malin R, Rantalaiho V, Huang XH et al. (1999) Association between M/L55-polymorphism of paraoxonase enzyme and oxidative DNA damage in patients with type 2 diabetes mellitus and in control subjects. Hum Genet 105:179-180

18. Trachtman H, Futterweit S, Maesaka J et al. (1995) Taurine ameliorates chronic streptozocin-induced diabetic nephropathy in rats. Am J Physiol 269:F429-F438

19. Lal MA, Korner A, Matsuo Y et al. (2000) Combined antioxidant and COMT inhibitor treatment reverses renal abnormalities in diabetic rats. Diabetes 49:1381-1389

20. Bursell SE, Clermont AC, Aiello LP (1999) High-dose vitamin E supplementation normalizes retinal blood flow and creatinine clearance in patients with type 1 diabetes. Diabetes Care 22:1245-1251

21. Morcos M, Borcea V, Isermann B et al. (2000) Effect of alpha-lipoic acid on the progression of endothelial cell damage and albuminuria in patients with diabetes mellitus: an exploratory study. Diabetes Res Clin Pract 52:175183 